九州大学学術情報リポジトリ

Kyushu University Institutional Repository

\title{
Thermal Convection in a Closed Water Body with Compound Cross Section
}

\author{
Hamagami, Kunihiko \\ Laboratory of Bioproduction and Environmental Information Sciences, Division of Bioproduction \\ and Environment Sciences, Kyushu University \\ Mori, Ken \\ Laboratory of Bioproduction and Environment Information Sciences, Division of Bioproduction \\ and Environment Information Sciences, Department of Bioproduction and Environmental Science, \\ Factory of Agriculture, Kyushu University \\ Hirai, Yasumaru \\ Laboratory of Bioproduction and Environment Information Sciences, Division of Bioproduction \\ and Environment Information Sciences, Department of Bioproduction and Environmental Science, \\ Factory of Agriculture, Kyushu University
}

https://doi.org/10.5109/17816

出版情報 : 九州大学大学院農学研究院紀要. 55 (1)，pp. 145-151，2010-02-26. Faculty of Agriculture, Kyushu University

バージョン：

権利関係 : 


\title{
Thermal Convection in a Closed Water Body with Compound Cross Section
}

\author{
Kunihiko HAMAGAMI ${ }^{1}$, Ken MORI* \\ and Yasumaru HIRAI
}

\author{
Laboratory of Bioproduction and Environment Information Sciences, Division of Bioproduction and \\ Environment Information Sciences, Department of Bioproduction and Environmental Science, \\ Factory of Agriculture, Kyusyu University, Fukuoka 812-8581, Japan \\ (Received November 13, 2009 and accepted November 19, 2009)
}

\begin{abstract}
In a closed water body with compound cross section, we examined the effects of horizontal convection based on the horizontal density difference on the characteristics of thermal convection generated by radiational cooling at water surface. An experiment for measuring water temperature revealed that the fluid exchange was done by the horizontal convection based on the density difference between deep and shallow parts. Moreover, a flow visualization experiment to investigate the characteristics of thermal, which are developmental factors in vertical convection, found that the generation frequency and the generation interval of thermals was higher in the case of compound cross section than the case of even depth. That is, it was understood that the change of the development characteristics of the mixed layer (vertical convection) based on the effect of horizontal convection occurred by the change of occurrence characteristics of the thermals.
\end{abstract}

\section{INTRODUCTION}

Water quality problems in closed water bodies such as agricultural ponds and creeks have received increasing attention in recent years. Because the amounts of inflow and outflow are generally low in closed water bodies, the circulation efficiency of water is low, too. Therefore, water with a lot of dissolved oxygen in the surface layer is not transported easily to the lower layer; the vicinity of the bottom layer includes little oxygen, and the water quality of the body becomes degraded. Since, water quality problems have been studied with closed water bodies, including eutrophication (Lap and Mori, 2007).

Major one of circulation in Closed water bodies is the day cycle, which consist of the formation of thermal stratification and the development of the mixed layer. Thermal stratification forms with heat transportation from the water surface by solar radiation in the daytime. Then, the development of the mixed layer is based on the mechanical disturbance by wind-induced flow (Mori et al., 1989) and on thermal disturbance by the cooling at water surface (Asaeda and Tamai, 1983). Two disturbances break down the thermal stratification, and the thickness of the thermal uniformed layer increases, in other words, the mixed layer.

Many studies have reported on thermal convection at high Rayleigh numbers, and it has been found that the flow structure becomes irregular as the Rayleigh number increases. This phenomenon occurs in the vicinity of the bottom where a silicone rubber heater heats the water, or near the water surface, where it is cooled; this was determined by Spangenberg and Rowland (1970), Foster (1969), and Katsaros (1978) through various methods. For the fluid dynamics based on the density difference,

${ }^{1}$ Laboratory of Bioproduction and Environmental Information Sciences, Division of Bioproduction and Environment Sciences, Kyushu University

* Corresponding author (E-mail: moriken@bpes.kyushu-u.ac.jp) there are two kinds of convection: vertical convection, based on the subsidence of a cold water mass (henceforth, "thermal") caused by the cooling at water surface (Asaeda et al., 1982), and horizontal convection, based on a horizontal unstable density caused by the depth difference or inhomogeneous cooling (Hamagami et al., 2008). The convection that originates in these density differences is thought to be one of the main flows in the closed water body. Therefore, it is important to clarify the characteristics of this density current to consider the water quality behavior of stagnated water body.

Here we aimed to clarify the effect of horizontal convection on the development characteristics of thermal convection in a closed water body with compound cross section. First, we conducted a experiment for measuring water temperature to examine the water temperature distribution in the deep, shallow, and step parts in order to clarify the characteristics of thermal stratification formation and the development of the mixed layer based on inhomogeneous heating and cooling by the depth difference in the water body. Moreover, we consider the pressure difference that exists between the deep part and the shallow part and a horizontal flow based on this difference in order to examine the scale of the horizontal convection. Next, we carried out a flow visualization experiment and consider about generation frequency, generation interval, and descent velocity in order to examine the characteristics of thermals which drives thermal convection by comparison with an experiment involving a case of even depth.

\section{METHODS AND RESULTS}

\section{Experimental equipment and methods}

Water temperature measurement

Figure 1 shows the outline of the test tank used in this research. It is made of an acrylic board $0.5 \mathrm{~cm}$ in thickness; the water surface area is $60 \times 20 \mathrm{~cm}$, with a depth of $30 \mathrm{~cm}$ in the deep part and $10 \mathrm{~m}$ in the shallow 
Table 1. The experimental conditions for the experiment for measuring water temperature

\begin{tabular}{|c|c|c|c|c|c|c|c|}
\hline \multirow{2}{*}{ Exp } & \multicolumn{2}{|c|}{ Wate depth $(\mathrm{cm})$} & \multirow{2}{*}{$\begin{array}{l}\text { Measurement } \\
\text { interval }(\mathrm{cm})\end{array}$} & \multirow{2}{*}{$\begin{array}{c}\text { Measurement } \\
\text { time interval (min) }\end{array}$} & \multicolumn{2}{|c|}{ Rayleigh number } & \multirow{2}{*}{$\begin{array}{c}\text { Amount of } \\
\text { evaporation }\left(\mathrm{cm}^{3} / \mathrm{h}\right)\end{array}$} \\
\hline & $H_{1}$ & $\mathrm{H}_{2}$ & & & Deep part & Shallow part & \\
\hline $1-1$ & 24 & 8 & 2 & 2.0 & $1.59 \times 10^{10}$ & $3.96 \times 10^{12}$ & 29.49 \\
\hline $1-2$ & 20 & 4 & $1(0 \sim 4 \mathrm{~cm}), 2(4 \sim 20 \mathrm{~cm})$ & 2.0 & $4.19 \times 10^{8}$ & $1.28 \times 10^{12}$ & 21.32 \\
\hline $1-3$ & 24 & 8 & 2 & 2.0 & $1.77 \times 10^{8}$ & $5.54 \times 10^{7}$ & 11.61 \\
\hline $1-4$ & 20 & 4 & $1(0 \sim 4 \mathrm{~cm}), 2(4 \sim 20 \mathrm{~cm})$ & 2.0 & $1.17 \times 10^{8}$ & $4.88 \times 10^{8}$ & 13.76 \\
\hline $2-1$ & 24 & 8 & $0.5(0 \sim 4 \mathrm{~cm}), 1(4 \sim 24 \mathrm{~cm})$ & 2.0 & $1.72 \times 10^{12}$ & $3.01 \times 10^{12}$ & 30.11 \\
\hline $2-2$ & 24 & 8 & $0.5(0 \sim 4 \mathrm{~cm}), 1(4 \sim 24 \mathrm{~cm})$ & 2.0 & $1.05 \times 10^{9}$ & $1.69 \times 10^{9}$ & 7.54 \\
\hline $2-3$ & 24 & 8 & $0.5(0 \sim 4 \mathrm{~cm}), 1(4 \sim 24 \mathrm{~cm})$ & 2.0 & $2.50 \times 10^{9}$ & $3.28 \times 10^{9}$ & 4.31 \\
\hline $3-1$ & 20 & 4 & 0.5 & 0.1 & $5.63 \times 10^{8}$ & $1.70 \times 10^{12}$ & 5.76 \\
\hline $3-2$ & 20 & 4 & 0.5 & 0.1 & $6.59 \times 10^{8}$ & $6.53 \times 10^{8}$ & 8.41 \\
\hline $3-3$ & 20 & 4 & 0.5 & 0.1 & $1.01 \times 10^{9}$ & $4.43 \times 10^{8}$ & 6.55 \\
\hline
\end{tabular}

part. Moreover, we conducted comparative experiments with a water tank that separated a shallow water body and a deep water body as a case of even depth. In addition, the cooling at water surface experiment examined for cases of uniformed and stratified thermal profiles. The experimental conditions are shown in Table 1. In Exp. 1, the water temperature distributions are measured by five sections in the case of compound cross section. In Exp. 2, the water temperature distribution is measured by one section in the deep water body and another in the shallow water body as a case of even depth. In Exp. 3, local water temperature distributions near the step part were measured by three sections in the case of compound cross section. The Rayleigh number is a dimensionless number that shows the scale of convection, and the flux type of Rayleigh number with the heat flux from the water surface $Q_{s}$ is shown in Eq. (1).

$$
R_{a}=\frac{\alpha g Q_{s} H^{4}}{\rho_{r} c \kappa^{2} v}
$$

where $\alpha$ is the coefficient of thermal expansion $\left(1 /{ }^{\circ} \mathrm{C}\right)$; $H$, water depth $(\mathrm{cm}) ; \kappa$, thermal diffusivity $\left(\mathrm{cm}^{2} / \mathrm{s}\right) ; v$, the coefficient of kinematic viscosity $\left(\mathrm{cm}^{2} / \mathrm{s}\right) ; Q_{s}$, heat flux $\left(\mathrm{J} / \mathrm{cm}^{2} \cdot \mathrm{s}\right) ; \rho_{r}$, reference density $\left(\mathrm{g} / \mathrm{cm}^{3}\right) ; c$, isopiestic specific heat $\left(\mathrm{J} / \mathrm{g} \cdot{ }^{\circ} \mathrm{C}\right)$; and $g$, gravitational acceleration $\left(\mathrm{cm} / \mathrm{s}^{2}\right)$. The parameters in Eq. (1) were defined as $\alpha=$ $2.5 \times 10^{-4}=2.5$ and $\kappa=1.4 \times 10^{-4}$.

To measure the amount of heat transportation from the water surface to the air (amount of evaporation), we measured air temperature, water temperature, humidity, and water level. The heat sources used to make the thermal stratification were an electrothermal board and an infrared ray lamp. These heat sources were set up at positions $30 \mathrm{~cm}$ above the water surface. The experiment was conducted indoors, and the air temperature was not controlled. Warm water (about $15^{\circ} \mathrm{C}$ higher than the air temperature at the start of the experiments) was used as an experimental fluid in the case of thermal uniformity. In the case of thermal stratification, the water was heated for 5 hours by the heat source, and then the cooling at water surface started. At that time, the bottom and sides of the water tank were covered with a styrene foam board $2 \mathrm{~cm}$ thick in order to satisfy adiabatic condition

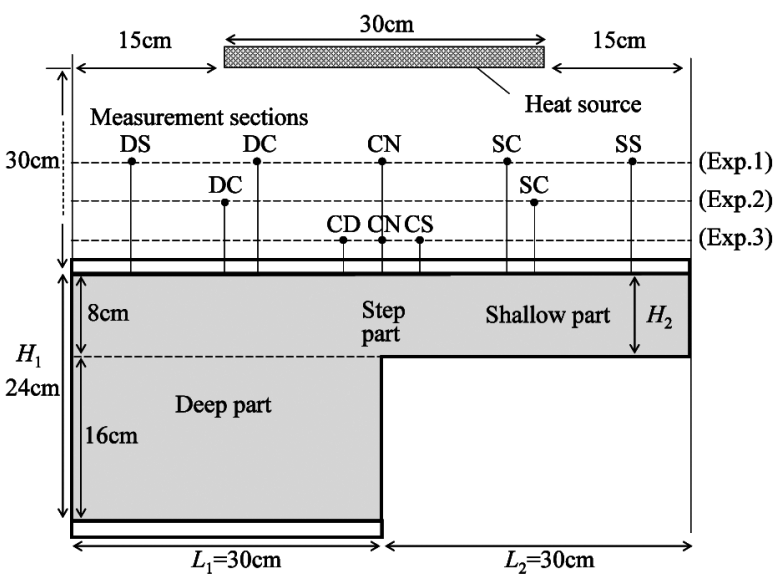

Fig. 1. The experimental equipment.

except the water surface. The water temperature was measured for 10 hours every 2 minutes in Exp. 1 and Exp. 2, and for 50 minutes every 6 seconds at a specific time zone within 10 hours in Exp. 3. Other measurement items except water temperature were measured for 10 hours every 2 minutes at a position $1 \mathrm{~m}$ away from the water tank.

\section{Flow visualization experiment}

Using both the same water tank and the same experimental procedure as the experiment for measuring water temperature, a flow visualization experiment was conducted with an aluminum powder method. We then examined the characteristics of thermals (generation frequency, generation interval, and descent velocity) by comparison between cases of even depth and compound cross section. A minute aluminum powder was evenly diffused in water, and a vertical section was made visible by a slit of light about $1 \mathrm{~cm}$ wide from the tank side in this experiment. The change over time was recorded from the front by a video camera. Additional measurement items were air temperature, humidity, and water temperature in the vicinity of the water surface. The experimental conditions of flow visualization are shown in Table 2. 
Table 2. The experimental conditions for the water temperature measurement

\begin{tabular}{cccccc}
\hline \multirow{2}{*}{ Case } & \multirow{2}{*}{$\begin{array}{c}\text { Shape of } \\
\text { water body }\end{array}$} & $\begin{array}{c}\text { Type of } \\
\text { fluid field }\end{array}$ & \multicolumn{2}{c}{ Water depth $(\mathrm{cm})$} & \multirow{2}{*}{ Rayleigh number } \\
\cline { 4 - 5 } & even depth & thermal uniformity & 24 & 8 & \multirow{2}{*}{$\begin{array}{l}H_{2} \\
\text { 4-1 }\end{array}$} \\
$4-2$ & uneven depths & thermal uniformity & 24 & 8 & $2.88 \times 10^{8}$ \\
$4-3$ & even depth & thermal stratification & 24 & 8 & $2.42 \times 10^{8}$ \\
$4-4$ & uneven depths & thermal stratification & 24 & 8 & $1.94 \times 10^{8}$ \\
\hline
\end{tabular}

\section{Experimental results and discussions}

Time change of water temperature distribution and heat flux at the water surface

Figure 2 shows the time change in the vertical distribution of water temperature in the case of thermal uniformity. Figure 2(a) shows the result in the case of even depth (Exp. 2-1), and Figure 2(b) shows the result in the case of compound cross section (Exp. 1-1). These figures show the vertical distribution of water temperature every hour after the cooling at water surface started in the central sections of the deep and shallow parts. From Fig. 2, it is understood that the water is cooled almost uniformly in vertical direction in all sections. Thus, the circulating flow based on thermal convection has occurred in the whole water tank. In Exp. 2-1, the shallow water body was cooled faster than the deep water body; by the end of the experiment, there was a difference of about $5{ }^{\circ} \mathrm{C}$. This is because of the difference in heat capacity due to the difference in depth between the two water bodies. On the other hand, in Exp. 1-1 the cooling rate was almost the same in the shallow and deep parts. This suggests that fluid is rapidly exchanged between the shallow and deep parts. That is, it is thought that the cooled water at the bottom of the shallow part flows into the deep part, and that the relatively warm water in the vicinity of the water surface of the deep part flows into the shallow part.

Figure 3 shows the time change in the vertical distribution of the water temperature during the period of the cooling at water surface in the case of thermal stratification. Figure 3(a) shows the result in the case of even depth (Exp. 2-3), and Figure 3(b) shows result in the case of compound cross section (Exp. 1-3). The inversion layer of the water temperature is formed by the decrement of water temperature in the vicinity of the water surface in either case. The water mass in the vicinity of the surface is transported downward by this unstable state of density, and a layer of uniform water temperature, that is, a mixed layer, develops. After then, the thickness of the mixed layer reaches the bottom, and the water temperature decreases uniformly in vertical direction. It is thought that the increase in water temperature in the lower layer, which the mixed layer does not reach, is caused by heat transmission from the upper layer.

Figure 4 shows the time change of the heat flux at the water surface. In this figure, the movement of heat from atmosphere to the water surface is defined as posi-

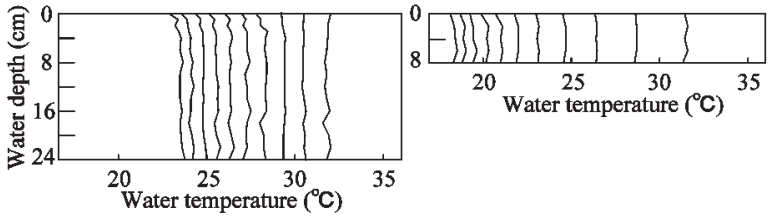

(a) The case of even depth (Exp.2-1)

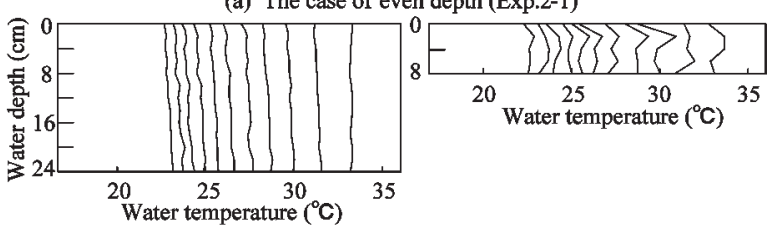

(b) The case of uneven depth (Exp.1-1)

Fig. 2. The time change of the water temperature in the case of thermal uniformity.

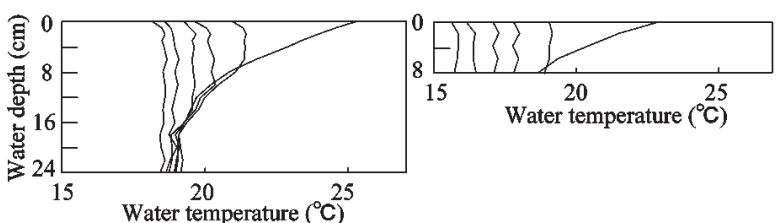

(a) The case of even depth (Exp.2-3)

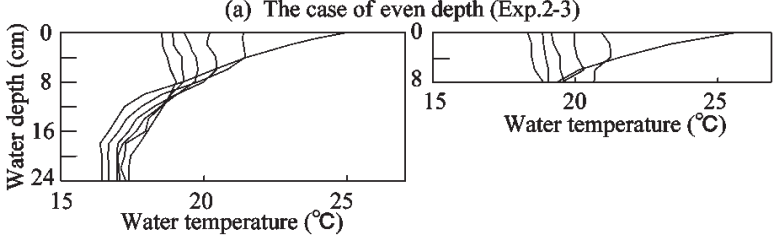

(b) The case of uneven depth (Exp.1-3)

Fig. 3. The time change of the water temperature in the case of thermal stratification.

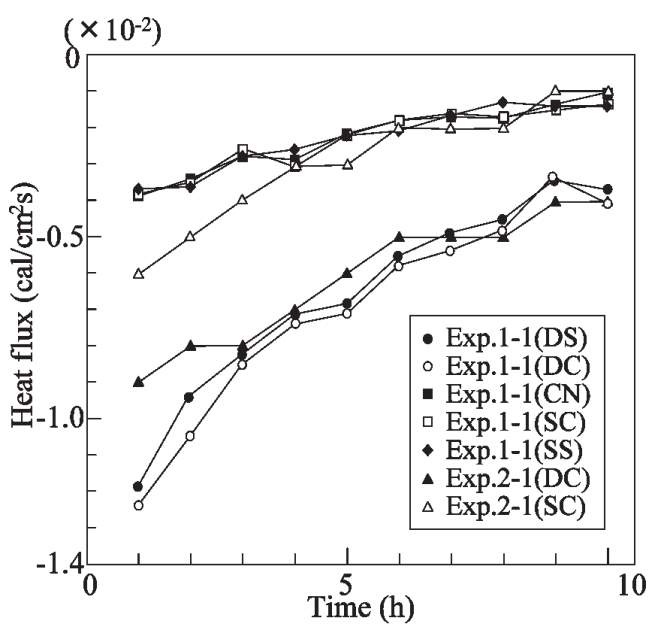

Fig. 4. The time change of the heat flux at the water surface. 
tive. When comparing Exp. 1-1 and Exp. 2-1 for the heat flux on the water surface at 1 hour after the beginning of the cooling, the heat flux in Exp. 1-1 is large in the deep part and small in the shallow part. The increase of heat flux means the increased loss of calories in the water column. The main factor of the loss is a density current based on the cooling at water surface in Exp. 2-1. That is, this difference may be attributable to the large contribution of water exchange based on the density current between the shallow part and the deep part in Exp. 1-1. Characteristics of the mixed layer

Figure 5 shows the decreasing velocity of the water temperature in the mixed layer in the case of even depth and in the case of compound cross section. In the shallow part with even water depth, the decreasing velocity of the water temperature in the mixed layer was large immediately after cooling started, and the velocity decreased rapidly as time passes. On the other hand, the decreasing velocity of the water temperature in the mixed layer was smaller in the deep part than in the shallow, and then the velocity decreased gradually. That is, there is a clear difference in the decreasing velocity of the water temperature in the mixed layer because depth (volume) differs between the two bodies. On the other hand, in the case of compound cross section there is no difference in the decreasing velocity of the water temperature in the mixed layer between the deep and shallow parts. The decreasing velocity decreases equally as time passes in both the deep and shallow parts. It is because water is exchanged by horizontal flow based on the difference in heat capacity.

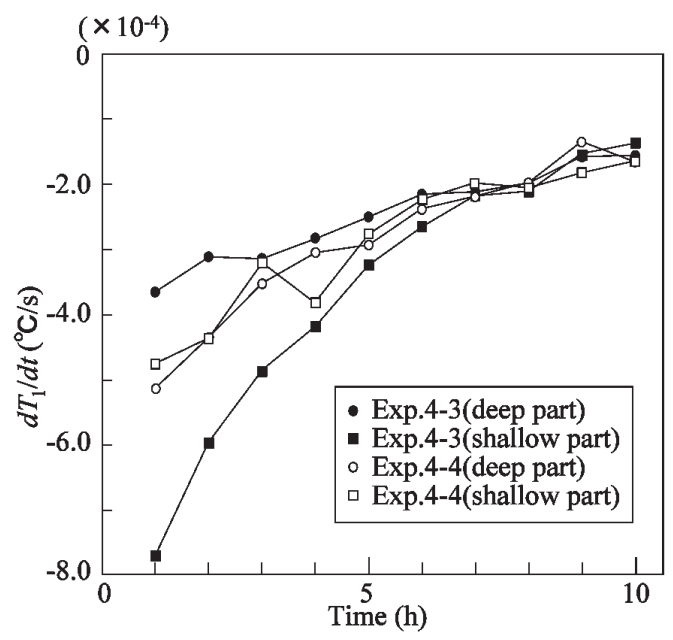

Fig. 5. The decreasing velocity of the water temperature in the mixed layer.

In the case of compound cross section, flow from the shallow to the deep part is generated because of the density difference between two parts. We deduce the flow velocity at this time bellow. Figure 6 shows the simple model of the density field. Figure 6(a) shows that water fields whose densities are $\rho_{1}$ and $\rho_{2}\left(\rho_{1} \leq \rho_{2}\right)$ are separated horizontally by the partition and also are in the quiescent condition. Removal of the partition from this state causes the horizontal flow by the density difference. The velocity of penetrative convection at this time is obtained from the viewpoint of energy preservation. That is, it is thought that in the fluid fields the density is different horizontally at first, as showed in Figure 6(a), and the fluid changes the state in which the density is vertically different, as showed in Figure 6(b), after the partition is removed. Assuming that the volume of each area is $V$, the potential energy at this time is

The potential energy to the bottom in the state shown in Figure 6(a):

$$
\frac{\rho_{1} V g H}{2}+\frac{\rho_{2} V g H}{2}=\frac{\left(\rho_{1}+\rho_{2}\right) V g H}{2}
$$

The potential energy to the bottom in the state shown in Figure 6(b):

$$
\frac{3 \rho_{1} V g H}{4}+\frac{\rho_{2} V g H}{4}=\frac{\left(3 \rho_{1}+\rho_{2}\right) V g H}{4}
$$

The difference is $\left(\rho_{2}-\rho_{1}\right) V g H / 4$. When the fluids in the upper and lower layers flow horizontally in opposite directions by the velocity $u$ like in Figure 6(b), the kinetic energy is $\rho_{1} V u^{2} / 2$ in the upper layer and $\rho_{2} V u^{2} / 2$ in the lower layer, and the total of these is $\left(\rho_{1}+\rho_{2}\right) V u^{2} / 2$. Assuming that the decreasing potential energy is converted to this kinetic energy,

$$
\frac{\rho_{2}-\rho_{1}}{4} \mathrm{VgH}=\frac{\rho_{2}+\rho_{1}}{2} V u^{2}
$$

Therefore, the velocity of penetrative convection is given by

$$
u=\sqrt{\varepsilon g h}
$$

where, $\varepsilon=\left(\rho_{2}-\rho_{1}\right) /\left\{\left(\rho_{2}+\rho_{1}\right) / 2\right\}$.

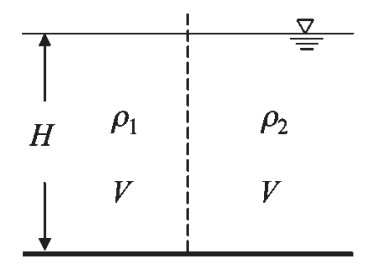

(a) Separated horizontally

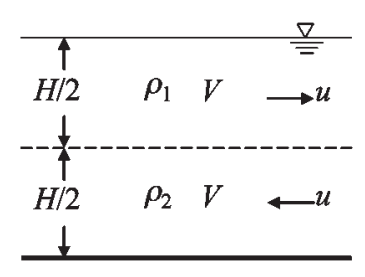

(b) Separated vertically
Fig. 6. The simple model of the density field.

Figure 7 shows the velocity of penetrative convection in Exp. 1-1 and Exp. 1-2. The velocity of the flow from the shallow part to the deep part (lower flow) is about $0.43(\mathrm{~cm} / \mathrm{s})$, and the velocity of the flow from the deep part to the shallow part (upper flow) is about 0.38 $(\mathrm{cm} / \mathrm{s})$ in Exp. 1-1. The righthand side of Eq. (5) is multiplied by the constant of proportionality in effect like $u$ $=\gamma \sqrt{\varepsilon g h}$. When we used the result of the flow visualization experiment under the same conditions as in Exp. $1-1$, the constant of proportion $\gamma$ became 0.92 in the lower flow and 0.78 in the upper flow.

The generation of a horizontal flow is based on the pressure difference which arises from density difference between the deep part and the shallow part. Then, to 


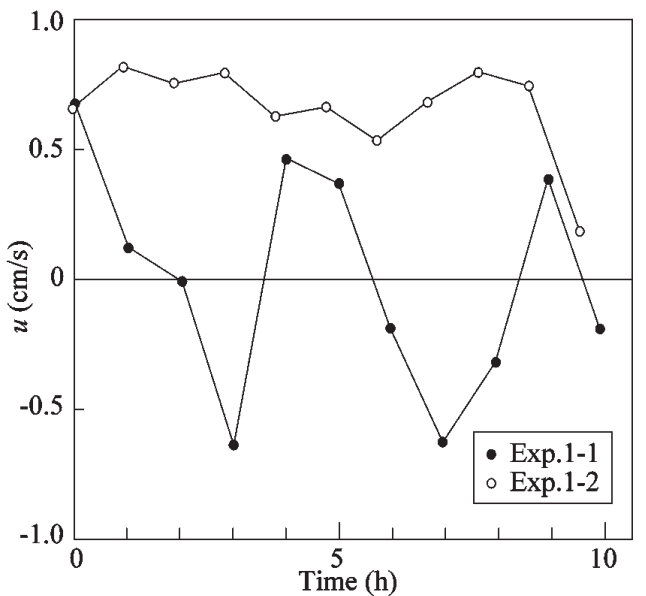

Fig. 7. The velocity of penetrative convection.

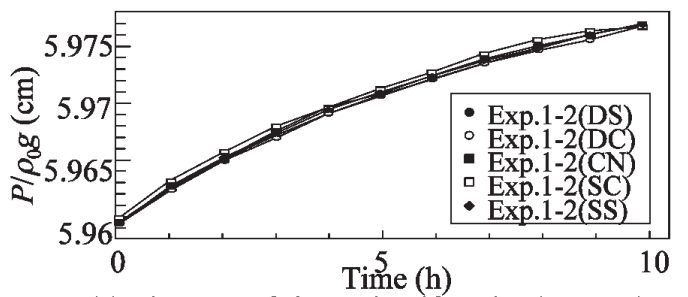

(a) The case of thermal uniformity (Exp.1-2)

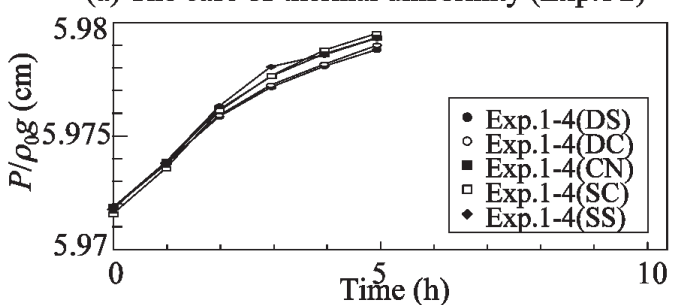

(a) The case of thermal stratification (Exp.1-4)

Fig. 8. The relationship in pressure between the deep and shallow parts.

examine the scale of the horizontal convection, we examined the relationship in static pressure between the deep and shallow parts in Figure 8. Figure 8(a) shows the result in the case of thermal uniformity (Exp. 1-2), and Figure 8(b) shows the result in the case of thermal stratification (Exp. 1-4). In Exp. 1-2, the pressure in the shallow part is higher than that in the deep part throughout the experiment time, though there is a small change in the pressure difference. On the other hand, in Exp. $1-4$, though the pressure in the deep part is higher than in the shallow part at the initial stage of cooling, the pressure in the shallow part falls back after 90 minutes. When there is a pressure difference in the water body, water flows from the high-pressure place to the lowpressure place to decrease the pressure difference. Therefore, it is understood that the characteristics of a horizontal flow from shallow to deep parts differ between cases of thermal uniformity and thermal stratification, and that the direction of a horizontal flow changes in the case of a stratification field, depending on the cooling stage.

Figure 9 shows the time change in the thickness of the mixed layer obtained from the vertical distribution of the water temperature. The thickness of the mixed layer $D$, which develops as a result of the cooling at water surface, is derived by the relation between the amount of heat transportation from the water surface and the amount of change of water temperature in the water body. Assuming that the calories lost from the water surface per unit time are $Q$, the relationship between the calories that the water body loses from time 0 to $t$ and the water temperature change is

$$
\int_{0}^{t} Q d t=\int_{0}^{H} \rho c\left(T-T_{s}\right) d z
$$

where $T$ is the water temperature at the water depth $z$ $\left({ }^{\circ} \mathrm{C}\right) ; T_{s}$, the water temperature at the water surface $\left({ }^{\circ} \mathrm{C}\right)$; and $\rho$, the water density. Thus, the thickness of the mixed layer $h$ is shown as

$$
h=\left(\frac{2 Q t}{\rho c \beta}\right)^{1 / 2}
$$

where $\beta$ is the water temperature gradient in the vertical direction. From this figure, it is understood that the relationship between the thickness of the mixed layer and the elapsed time in this experiment complies with Eq. (7).

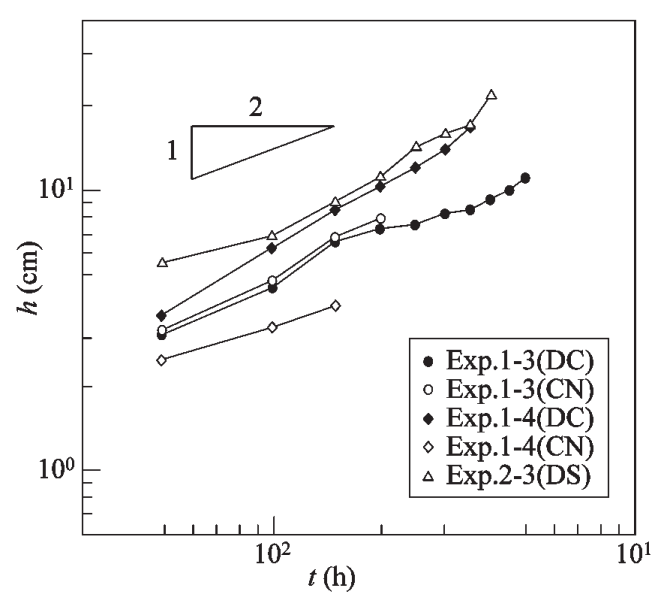

Fig. 9. The time change in the thickness of the mixed layer.

Our experiment in water temperature measurement to examine the characteristics of mixed layers in water bodies with compound cross section shows that the horizontal convection based on the density difference between the deep and shallow parts affects significantly the time change of the thickness of the mixed layer. The development of the mixed layer is caused by the many actions of small thermals. We therefore examined the characteristics of thermals in order to consider in detail the influence of the horizontal convection on the vertical convection.

\section{Characteristics of thermal}

In this chapter, the occurrence characteristics of thermals (generation frequency, generation interval, and descent velocity) are examined in a flow visualization experiment in comparison with the results of the experi- 
ment in the case of even depth. In thermal convection based on the cooling at water surface, vertical convection is formed by a thermal movement settling from the vicinity of the water surface and its compensation flow. That is, it is thought that the generation characteristics of thermals change by the influence of horizontal convection. Then, through a flow visualization experiment using an aluminum powder method, we examined the influence of the horizontal convection generated in the water body with compound cross section on the occurrence characteristics of thermals.

When we consider the cooling at water surface in an immovable thermal stratification, the transportation of heat is based on heat conduction only at the beginning of cooling, and thermal convection is not caused. When the cooling process progresses and the water near the water surface reaches an unstable density, a thermal is formed by the subsidence of the cold water mass, thus establishing the shape involved in the descent by the shearing stress from surrounding fluid. From this, it is thought that the generation interval and the generation frequency of a thermal are decided by the stability of the water surface layer. In the result of the stability analysis by Chandrasekhar (1961), the limit of flux types of Rayleigh number that become unstable is $R_{a c} \cong 1100$, and the relationship between the horizontal scale $L_{v}$ and the reference scale in the vertical direction $d$ is $d=2.68 L_{v} / 2 \tilde{\pi}$. The reference scale $d$ is obtained as $d=\left(R_{a c} \rho_{r} c \kappa^{2} v\right)$ $\left.\alpha g Q_{s}\right)^{1 / 4}$ from the definitional equation of $R_{a}$ (Eq. (1)), so that the following relationship is almost proven even if there is a difference between the timepoint of the stabilization of density breakdown and the timepoint where the thermal is generated.

$$
L_{v} \propto\left(R_{a c} \rho_{r} c \kappa^{2} v / \alpha g Q_{s}\right)^{1 / 4}
$$

In addition, assuming that heat is transported by heat conduction until the unstable state is reached, the time until instability is reached $T_{v}$ is calculated from $d=\sqrt{\kappa T_{v}}$.

$$
T_{v}=\left(R_{a c} \rho_{r} c v / \alpha g Q_{s}\right)^{1 / 2}
$$

Thus, the generation frequency of the thermal generated at the unit area and the unit time $f_{v}$ is obtained by

$$
f_{v}=\frac{1}{T_{v} L_{v}^{2}}=m \cdot R_{a} \frac{\kappa}{d^{4}}
$$

where $m\left(=1.0 \times 10^{-4}\right)$ is a constant of proportion (Asaeda et al., 1982). From Eq. (10), it is understood that the generation frequency of a thermal does not depend on depth. Moreover, the average descent velocity of thermal $w_{v}$ is decided from the heat flux at the water surface, and it is given by the dimension analysis as

$$
w_{v} \propto\left(\alpha g Q_{s} d / \rho_{r} c\right)^{1 / 3}
$$

Figure 10 shows the generation frequency of a thermal in the flow visualization experiment, Exp. 4. The experimental result is an average value for an observation period of about 30 minutes. For the observation, the number of thermals was counted when a new thermal was generated after the previous thermal had subsided completely. From this figure, it is understood that the experimental result in Exp. 4-2 (the case of even depth) complies with Eq. (11). The generation frequency of thermals is higher in the case of compound cross section than in the case of even depth, though the relationship to the Rayleigh number has the same tendency as that in the case of even depth. The results also show that thermals are frequently generated by an unstable density in the case of compound cross section.

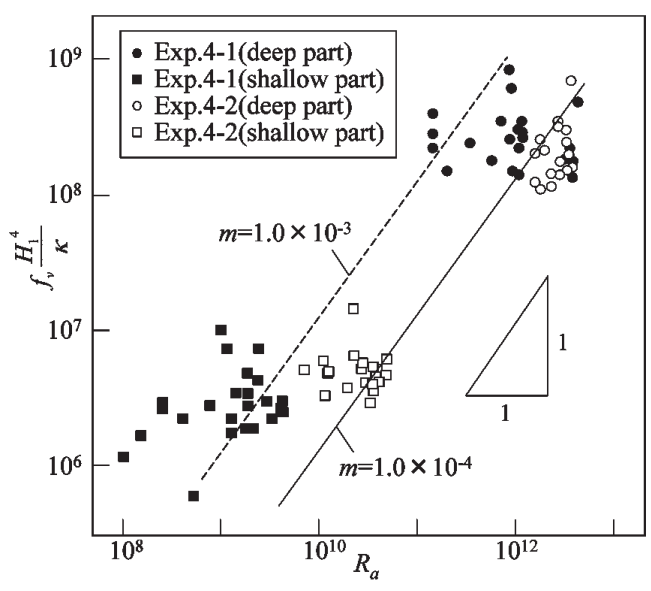

Fig. 10. The generation frequency of thermals.

Figure 11 shows the generation interval of thermals in the flow visualization experiment, Exp. 4. The generation interval of thermals is defined as the length of the water surface divided by the number of thermals, because the generation interval of thermals was almost equal horizontally. This figure shows that the experimental result complies with Eq. (8). When comparing between the case of even depth and compound cross section, there is a tendency toward a large generation interval of thermals in the case of compound cross section. That is, it is thought that the generation interval of thermals increases because convection cells develop more rapidly when the generation frequency of thermals increases.

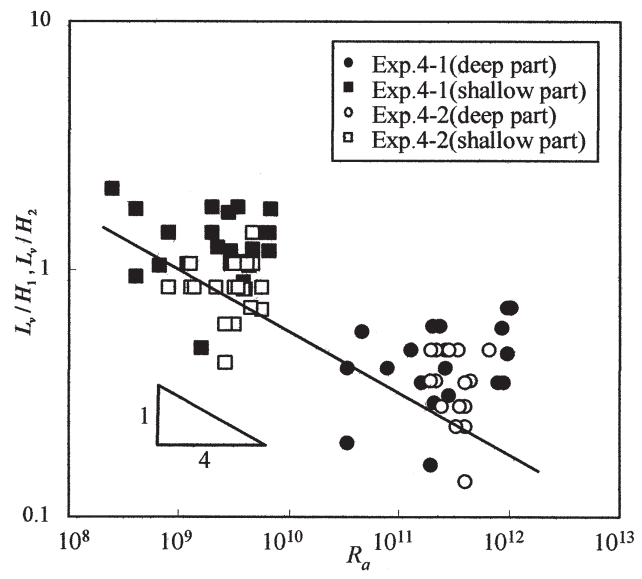

Fig. 11. The generation interval of thermals. 
Figure 12 shows the descent velocity of thermal $w_{v}$ in the flow visualization experiment, Exp. 4. The descent velocity was defined as the velocity by which the thermal descended in depth by $2-4 \mathrm{~cm}$. From this figure, it is understood that the experimental result complies with Eq. (11). When we compare the cases of even depth and compound cross section, no clear difference is seen in the tendency, and it is understood that the descent velocity of a thermal can be expressed by Eq. (11) approximately in either case. That is, it is clarified that the descent velocity of a thermal in the vicinity of the water surface is the same in the case of even depth as in the case of compound cross section.

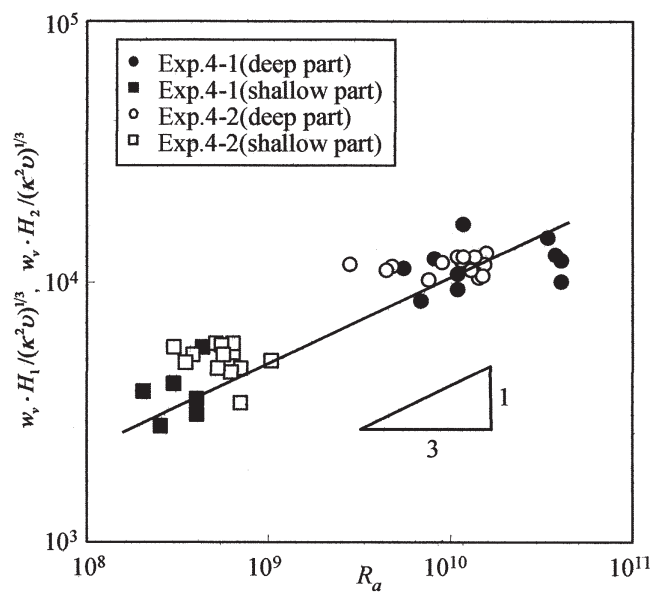

Fig. 12. The descent velocity of thermals.

These results suggest that the generation frequency and the generation interval of thermals were higher in the case of compound cross section than in the case of even depth. It is shown also that the horizontal convection based on the density difference between the deep part and the shallow part affects the generation characteristics of thermals. That is, the change in the development characteristics of the mixed layer (vertical convection) based on the effect of the horizontal convection was caused by the change in the occurrence characteristics of thermals.

\section{CONCLUSION}

In this study, the effects of horizontal convection based on the horizontal density difference on thermal convection based on the cooling at water surface were examined in a closed water body with compound cross section, and the following results were obtained.

(1) An experiment for measuring water temperature revealed that the characteristics of vertical convection based on the cooling at water surface were affected by horizontal convection based on the density difference between deep and shallow parts.

(2) A flow visualization experiment to investigate the occurrence of thermal characteristics, which are developmental factors in vertical convection, found that the generation frequency and the generation interval of thermals was higher in the case of compound cross section than in the case of even depth. That is, it was understood that the change in the development characteristics of the mixed layer based on the effect of horizontal convection was caused by the change in the occurrence characteristics of the thermals.

\section{ACKNOWLEDGEMENTS}

The authors wish to thank Laboratory of Drainage and Water Environment, Division of Regional Environmental Science, Department of Bioproduction Environment Science, Faculty of Agriculture, Kyushu University for the convenience in the conducting the experiment.

\section{REFERENCES}

Asaeda, T., S. Ikeda and Y. Ninomiya 1982 Coherent Structures in Thermal Convection. Proceedings of the Japanese Conference on Hydraulics, 26: 447-452

Asaeda, T., N. Tamai, 1983 On characteristics of plumes observed in the thermal convection with the large Rayleigh number. Proceedings of the Japan Society of Civil Engineers., 336 65-73

Chandrasekhar, S. 1961 Hydrodynamic and Hydromagnetic Stability. Qxford Univ. Press

Foster,T. D. 1969 Onset of manifest convection in a layer of fluid with a time-dependent surface temperature. Phys. Fluids., 12: $2482-2487$

Katsaros, K. B. 1978 Turbulent free convection in fresh and salt water: some characteristics received by visualization. J. Phys. Ocean., 3: 613-626

Lap, B. Q. and K. Mori, 2007 A two-dimensional numerical mode of wind-induced flow and water quality in closed water bodies. Paddy Water Environ., 5(1): 29-40

Mori K., Y. Tohara, S. Shikasyo. K. Hiramatsu. O. Kato and H. Cho 1989 The Diurnal Mixed Layer and Its Influence on the Velocity Field (at Lake Kasumigaura). Trans. of JSIDRE, 144: $75-84$

Spangenberg, W. G. and W. R. Rowland 1970 Convective circulation in water induced by evaporating cooling. Phys. Fluids., 4: $793-800$ 